\title{
Trends in antibiotic resistance among bacteria isolated from blood cultures using a large private laboratory network data in India: 2008-2014
}

\author{
S Gandra ${ }^{1 *}$, N Mojica ${ }^{1}$, A Ashok$^{2}$, BR Das $^{3}$, R Laxminarayan $^{1,4,5}$ \\ From 3rd International Conference on Prevention and Infection Control (ICPIC 2015) \\ Geneva, Switzerland. 16-19 June 2015
}

\section{Introduction}

Antimicrobial resistance surveillance is essential to track changes in microbial populations, estimate the magnitude of the problem and to design and evaluate interventions. However, there is no national level information on resistance among bacteria causing bloodstream infections in India.

\section{Objectives}

The purpose of the study is to examine the extent and trends of antibiotic resistance among bacteria isolated in blood cultures from January 1, 2008 to December 31, 2014.

\section{Methods}

Antibiotic susceptibility data were obtained from more than 275 microbiology laboratories spread across 24 states in India which are part of a large private laboratory network. We retrospectively examined trends of resistance in pathogenic bacteria (Salmonella typhi/paratyphi, Escherichia coli, Klebsiellaspecies, Staphylococcus aureus, Acinetobacter species and Pseudomonas aeruginosa) isolated from blood cultures for years 2008-2014.

\section{Results}

A total of 136,915 unique blood cultures were obtained over the seven years period and total positive cultures were 17,494 (13\%). The breakdown of the organisms by frequency of isolation include: coagulase negative staphylococcus (4488, 26\%), Salmonella sp. (typhi/paratyphi) (3202, 18\%), E.coli (2191, 13\%), Klebsiella sp. (1401, 8\%), S. aureus (1053, 6\%), Acinetobacter sp. (1021, 6\%) Pseudomonas sp. (794, 5\%) and others (3344, 19\%). Ciprofloxacin resistance in Salmonella sp. increased from 13\% in 2008 to $22 \%$ in 2014. Third generation cephalosporin resistance in E.coli was $74 \%$ in 2008 and increased to $80 \%$ in 2014 and in Klebsiella sp., it was $94 \%$ in 2008 and decreased to $80 \%$ in 2014. Carbapenem resistance in Klebsiella sp. was 22\% in 2008 and increased to $60 \%$ in 2014 and in E.coli, was 7\% in 2008 and increased to $12 \%$ in 2014. Carbapenem resistance in Acinetobacter sp. was $73 \%$ in 2008 and decreased to $69 \%$ in 2014 and in P. aeruginosa it was $55 \%$ in 2008 and decreased to $37 \%$ in 2014 . Methicillin resistance $S$. aureus increased from $50 \%$ in 2008 to $55 \%$ in 2014 .

\section{Conclusion}

Very high rates of resistance were observed to frontline and last-resort antibiotics among bacteria isolated from blood cultures in India.

\section{Disclosure of interest}

None declared.

\section{Authors' details \\ ${ }^{1}$ Center for Disease Dynamics, Economics \& Policy, New Delhi, India. ${ }^{2}$ Center for Disease Dynamics, Economics \& Policy, Washington DC, USA. ${ }^{3}$ SRL Diagnostics Ltd., Mumbai, India. ${ }^{4}$ Public Health Foundation of India, Gurgaon, India. ${ }^{5}$ Princeton Environmental Institute, Princeton, NJ, USA.}

Published: 16 June 2015

doi:10.1186/2047-2994-4-S1-O42

Cite this article as: Gandra et al:: Trends in antibiotic resistance among bacteria isolated from blood cultures using a large private laboratory network data in India: 2008-2014. Antimicrobial Resistance and Infection Control 2015 4(Suppl 1):042.

${ }^{1}$ Center for Disease Dynamics, Economics \& Policy, New Delhi, India Full list of author information is available at the end of the article 